\title{
A relationship exists between replicative senescence and cardiovascular health
}

\author{
Maria E Karavassilis ${ }^{1}$ and Richard Faragher ${ }^{2^{*}}$
}

\begin{abstract}
A growing body of evidence demonstrates that the accumulation of senescent cells is a plausible ageing mechanism. It has been proposed that the senescence of vascular cells plays a causal role in the development of cardiovascular pathologies. A key prediction arising from this hypothesis is that cultures of cells derived from donors with cardiovascular disease will show reduced in vitro replicative capacities compared to those derived from disease-free controls. Accordingly, we carried out a formal review of the relationship among donor age, cardiovascular health status and maximum population doubling level attained in vitro by cultures of vascular smooth muscle and endothelial cells. Data were available to us on a total of 202 independent cell cultures. An inverse relationship was found to exist between replicative capacity and donor age in both endothelial and vascular smooth muscle cells. Cultures derived from donors with cardiovascular disease showed a lower overall replicative potential than age-matched healthy controls. In general the replicative potential at the start of the lifespan was found to be higher in those individuals without disease than those with disease and the difference in average cumulative population doublings (CPDs) in age-matched individuals in the two groups remained roughly constant throughout the lifetime. These results are consistent with the model in which the inherited replicative capacity of vascular cells is a stronger determinant of the onset of cardiovascular disease later in life, than wear-and-tear throughout the life course.
\end{abstract}

Keywords: Replicative senescence, Vascular endothelial cells, Vascular smooth muscle cells, Ageing, Atherosclerosis, Hypertension

\section{Review}

Introduction

\section{The purpose of this article}

A detailed understanding of the mechanisms underlying cardiovascular disease has important implications for the reduction of mortality and morbidity in the aged population. For largely historical reasons, the majority of studies of replicative senescence have focused on human fibroblasts, as it was in these cell types that the phenomenon of cellular senescence was first reported. Unfortunately, this has produced a distortion of the evidence base, which renders a detailed understanding on the relationship replicative senescence and health status problematic. This is because very few age-associated pathologies are directly attributable to the dermal layer

\footnotetext{
* Correspondence: R.G.A.Faragher@brighton.ac.uk

${ }^{2}$ School of Pharmacy and Biomolecular Sciences, University of Brighton, Huxley Building, Lewes Road, Brighton BN2 4GJ, UK

Full list of author information is available at the end of the article
}

of the skin (or other fibroblastoid tissue layers, such as the corneal stroma).

However, a number of cell types exist in which a direct link between the senescent phenotype and age-associated disease is potentially easier to interrogate. Endothelial cell (EC) and vascular smooth muscle cell (VSMC) senescence in particular has been linked to the development of cardiovascular disease, specifically atherosclerosis [1-3]. The senescent phenotype has been proposed to result in an impaired ability to replace damaged or lost cells or to produce altered tissue microenvironments within the vessel [4]. Accordingly we reviewed the available data on proliferative capacity, donor age and cardiovascular disease status with regard to these cell types, in order to produce a meta-dataset spanning a range of age groups and cardiovascular disease states, allowing provisional conclusions to be drawn.

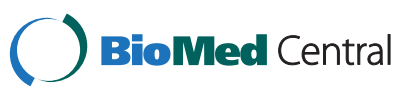




\section{The cell hypothesis of ageing}

The hypothesis that the progressive accumulation of senescent cells with tissue turnover during life plays a causal role in ageing was first proposed more than 40 years ago and has gained increasing credibility in recent years [5]. Classic studies in this area include that of Martin et al. [6], who reported a reduction of approximately two population doublings per decade in fibroblasts derived from individuals aged from new-born to a hundred years, and that of Schneider and Mitsui [7], who demonstrated a reduced average fibroblast migration rate, lower replication rate and saturation density at confluence, in fibroblast cultures derived from older individuals compared to their younger counterparts.

However, several studies using fibroblast cultures have demonstrated no statistically significant decline in replicative potential with age $[8,9]$. One explanation for lack of correlation is the exclusive selection of healthy donors. Because senescent cells are highly likely to be causal agents of age-related disease [5], it follows that these may be absent in a study population comprising only healthy donors.

Maier and Westendorp (2008) [10] proposed that the relationship between senescence and ageing is attributable to publication bias, and is absent in studies comprising large sample populations. However, this work contains questionable methodological aspects, the major constraint being the use of data derived from the fibroblast literature. Skin samples are simply not directly reflective of the pathological mechanism underlying Alzheimer's disease.

There are literature reports where the proliferative capacity of fibroblasts from centenarians is not significantly reduced compared to those from younger donors [11]. This may reflect the fact that fibroblast replicative capacity in culture is not representative of the key organ systems in vivo.

The notion that senescence is a causal process driving at least some aspects of ageing is undeniable, as senescent cells have been found to be present and accumulate in tissue in vivo as a function of organismal age $[12,13]$. Among the best evidence of this is provided by a study where the number of mitotic cells in the proliferative region of murine lens epithelium was seen to decline with the age of the organism, with a concurrent increase in senescent cells [14].

The presence of senescence-associated markers at sites of pathology supports the relationship between replicative senescence and age-related disease. Using senescence-associated beta-galactosidase, senescent endothelial cells have been found to accumulate after repeated balloon endothelial denudation of the rabbit carotid artery [15]. It has also been demonstrated that telomeres in the endothelium shorten with age and this is more pronounced in atherosclerosisprone areas [14-16]. Moreover, cultures of vascular smooth muscle cells derived from plaques have been reported to show a reduced proliferative capacity [17].

The senescent phenotype of vascular cells is not inconsistent with the potential to cause cardiovascular disease. Senescent endothelial cells in vitro have been found to over-express proteins characteristic of the proinflammatory and pro-thrombotic phenotype of the endothelium in human atherosclerosis, including IL1alpha, ICAM-1 and PAI-1 [18-20]. Burton et al. (2009) demonstrated that key genes known to be up-regulated in atherosclerotic plaques are also highly up-regulated in senescent VSMCs. Most importantly, it was found that senescent VSMCs adopt a phenotype which contributes to the pathogenesis of vascular calcification, characterized by the expression of genes associated with vascular calcification, namely matrix Gla protein, bone morphogenetic protein-2, osteoprotegerin, osteopontin and decorin [21].

\section{Hypothesis}

The relationship between senescence and disease state throughout the lifetime can be modeled in three ways. In the first hypothesis, all individuals are expected to show similar inherited replicative capacities at the start of their lifespan. In subjects without cardiovascular disease, senescence is assumed to be the consequence of lifelong reparative cell divisions with advancing age [22-24]. In subjects with cardiovascular disease, senescence may be thought to represent an acceleration of the biological ageing process, triggered by exposure to mitotic stress, oxidative stress or DNA damage, independent of chronological age [25-27]. Those more prone to developing disease are, therefore, expected to exhaust their replicative potential at an accelerated rate compared to healthy individuals. The expected graphical relationship shows the trend lines of diseased and healthy individuals originating on the same point on the Y-axis, from which they diverge, with the line representing diseased individuals showing a steeper slope (Figure 1).

It may otherwise be the case that replicative capacity always declines consistently with chronological age, regardless of health status (Figure 2). In this case, the expected graphical relationship should show that both trend lines coincide throughout the organism's lifespan.

An alternative hypothesis is that individuals differ in inherited replicative capacity at the start of their lifespan, but exhaust their replicative potential at a similar rate. Those more prone to developing disease are expected to start off with a lower replicative capacity (Figure 3 ). The expected graphical relationship should show the trend line corresponding to diseased individuals to start out at a lower point on the $y$-axis compared to non-diseased 
individuals, after which point the trend lines decline at similar rates, appearing to be parallel.

Individual differences in replicative capacity may be attributed to variations of inherited telomere lengths, as has been suggested in several studies. In one report, blood telomere lengths were found to range from 5.10 $\mathrm{kb}$ in young men from Italy to $18.64 \mathrm{~kb}$ in young men from Belgium, showing a greater than three-fold difference across populations [28]. In another study, individuals of African-ancestry in the USA tended to show 10\% longer blood telomere lengths compared to those of European ancestry [29]. The large variation of telomere lengths observed in adults was suggested to relate to adaptive evolution, differences in early life experience or growth [30], exposure to stress throughout the lifespan [31] and, most pertinently in this case, varying paternal ages at reproduction. Kimura et al. [32] investigated the relationship between telomere length in sperm from young (30 years) and older (50+ years) donors with mean leukocyte telomere length in offspring (in adult ages); they demonstrated that a subset of sperm from older men had elongated telomeres, which corresponded to a positive relationship between paternal age at reproduction and offspring telomere length. The variation of telomere length in embryo-derived cells appears not to have been studied in detail.

Certainly, vascular cells with inherited short telomeres that are also exposed to considerable amounts of stress throughout the life course should show a much lower replicative potential compared to healthy individuals. The most likely hypothesis is, therefore, based on the notion that most disease states are multi-factorial in nature. When combining data from many individuals, it is expected that the relationship between replicative capacity and ageing in diseased individuals will show an overall lower cumulative population doubling (CPD) potential compared to healthy controls, as well as an accelerated rate of decrease of CPD.

\section{Materials and methods \\ Data mining}

We mined the available peer-reviewed literature (principally using PubMed and relevant electronic journals directly). Combinations of search terms used included: 'endothelium,' 'vascular smooth muscle cells,' 'senescence,' 'cumulative population doublings', 'in vitro', 'passage' and 'donor age'. The search covered over 10,000 research articles. When available, the following information was obtained: age of donor, replicative capacity in vitro, vessel of origin, gender, incidence of cardiovascularrelated disease and definition of replicative senescence. Articles were excluded if replicative capacity in vitro was recorded in such a way that $\mathrm{CPD}$ could not be accurately deduced. Ultimately, 12 research articles were used, published between 1978 and 2008.

\section{Replicative capacity and health status}

Studies were included in this review if donor age and replicative capacity of EC or VSMC were recorded. If not given, the CPD value was extrapolated from maximum passages achieved (given the cell culture split ratio) or approximated from scatter plots. If health status was not mentioned, it was assumed that the donors did not suffer any cardiovascular-related diseases.

\section{Statistical analysis}

To assess the strength of the correlation between in vitro replicative capacity and age of donor, regression analyses were carried out for each cell type, as well as sample populations with/without vascular-related diseases separately. For endothelial cells, graphs including all data points as well as graphs excluding human umbilical vein endothelial cells (HUVECs) are shown, as HUVEC samples show a very large scatter, which affected the trend lines. The mean decade CPD was plotted against donor decade age, to balance out any effects from possible confounding variables.

The mean rate of change of CPD with respect to age (change in CPD per year) was calculated for the graphs of: 'all CPD values versus donor age,' 'mean CPD versus donor age decade' and for the graphs of 'CPD versus donor age' in those with or without vascular-related diseases shown separately.

To assess the contribution of confounding factors, scatter plots were drawn to visualize clustering of data points, per vessel type and per gender.

T-tests were performed to assess whether the difference in mean CPD for adjacent decades as well as decades at either extreme, could be attributed to chance. A T-test was also carried out comparing CPD of male versus female donors for each cell type.

\section{Results}

\section{Replicative capacity in vitro versus age of donor}

The linear regression line shows a clear inverse relationship between replicative capacity in vitro and donor age, indicating a negative correlation for VSMC and EC. For both cell types, it is evident from the graphs that in the $50+$ age group there are a larger proportion of points with low CPD values, that is, below 10 CPD in VSMCs and below 20 CPD in ECs.

When the decade mean CPD is plotted against donor age, a negative correlation is seen for both cell types, with $R=-0.866$ for VSMC (Figure 4 ), $R=-0.733$ for EC (Figure 5).

T-tests were carried out between datasets for adjacent decades. The $P$-values for the comparisons between 


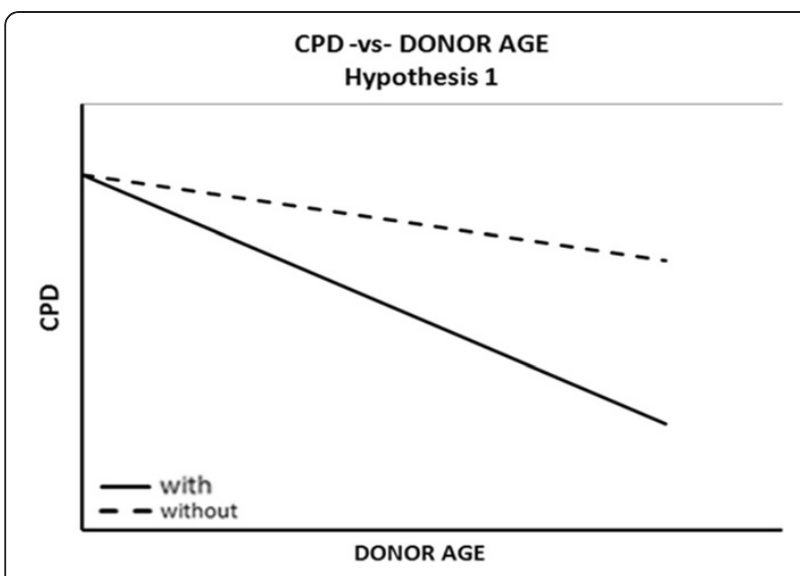

Figure 1 Hypothesis 1: diseased and healthy individuals start with similar replicative capacities. Replicative capacity is exhausted at a faster rate in diseased individuals.

adjacent decades in both cell types (with the exception of decades 4 and 5 in EC), indicate that one cannot say with certainty that the observed difference for adjacent decades cannot be attributed to chance (Table 1). The $P$-values comparing the first and eighth decades indicate that the differences are highly significant. The same conclusion was drawn when comparing the first and last two and three decades in VSMC and EC respectively. Thus, based on the available dataset, although it is not strongly evidenced that small age differences show a decrease in CPDs, a large age difference is clearly associated with a substantial decrease in CPD.

The mean rate of change in CPD with respect to age was also evaluated by calculating the slope of the linear regression line (Table 2). Table 3 shows the mean rate of change in CPD with respect to age, per publication. One can see that VSMC shows a lower rate of decrease per year $(-0.19)$ compared to EC $(-0.44)$ and similar rate of decrease when excluding HUVECs (-0.15). Looking at the mean decade CPD with respect to age decade, both cell types gave the same value $(-0.19)$ of rate of change.

\section{Replicative capacity in vitro versus age of donor with respect to cardiovascular-related diseases}

The data points from donors with cardiovascular-related diseases show a negative correlation with age, with $\mathrm{R}=-0.511$ in VSMCs (Figure 6) and $\mathrm{R}=-0.24$ in EC (Figure 7). The data points from donors without cardiovascular related disease also show a negative but slightly weaker correlation with age $\mathrm{R}=-0.411$ in VSMC (Figure 6) and much stronger correlation with age $\mathrm{R}=-0.708$ in EC (Figure 7) and -0.799 when excluding HUVEC (Figure 8). It is useful to exclude HUVEC data points as they show a very wide scatter. Comparing the trend lines visually, it is clear that on average, CPDs are higher in those without cardiovascular-related disease compared to those with the disease for both cell types (when excluding HUVEC).

The T-tests between the sample populations with or without cardiovascular-related diseases show extremely small $P$-values of 3.89E-11 for VSMC and 1.21E-14 for EC and 1.11E-2 for EC, excluding HUVEC, indicating with a high degree of confidence that the observed difference between the means of the two data sets is not attributed to chance.

The change in CPD with respect to age in those without cardiovascular-related disease (Table 2) shows a

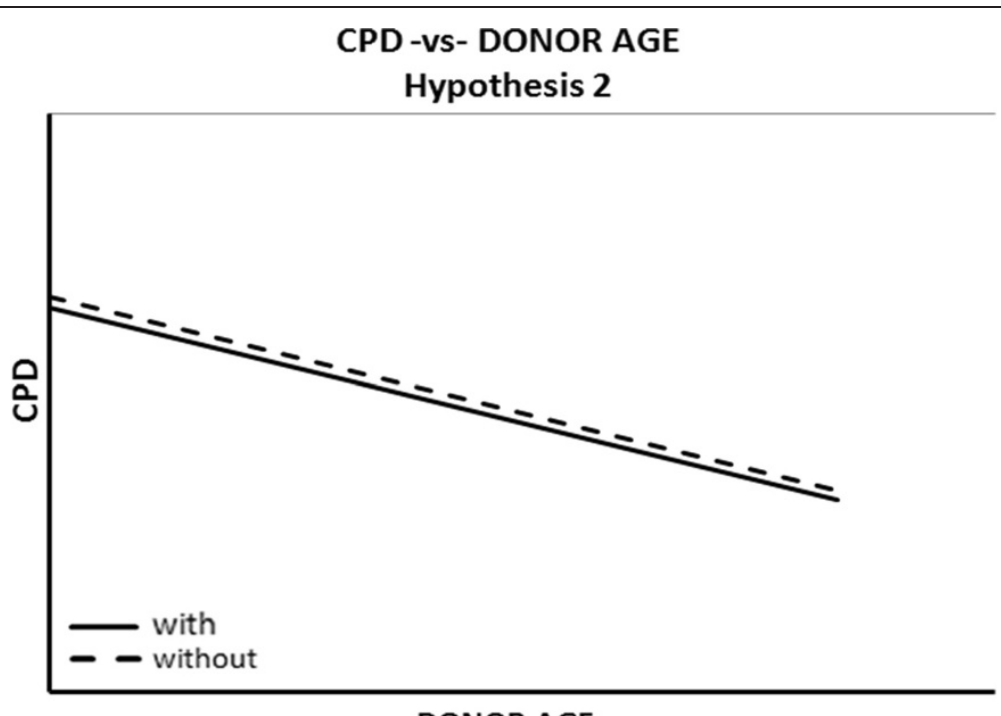

DONOR AGE

Figure 2 Hypothesis 2: diseased and healthy individuals coincide in terms of replicative capacity throughout lifespan. 


\section{CPD -vs- DONOR AGE \\ Hypothesis 3}

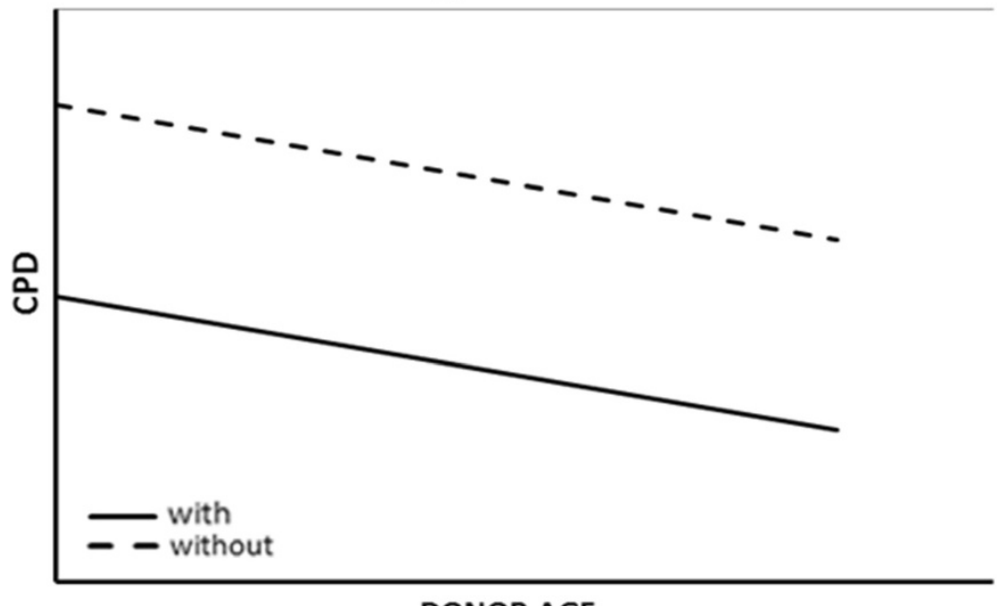

DONOR AGE

Figure 3 Hypothesis 3: diseased and healthy individuals start off with different replicative capacities. Replicative capacity is exhausted at similar rates.

slightly lower rate of decrease per year compared to those without cardiovascular-related disease, in both cell types, that is, -0.14 in ECs from donors with cardiovascular-related diseases, -0.47 in ECs without cardiovascular-related diseases, -0.16 in ECs without cardiovascular-related diseases, excluding HUVECs, -0.17 in VSMCs from donors with cardiovascular-related diseases, -0.2 from donors without cardiovascular-related diseases.

Scatter plots were drawn to assess the effects of vessel type and gender on EC and VSMC replicative capacity (Figures 9, 10, 11, 12). Overall, the data-points are seen to be equally spread in both cell types, regardless of gender or vessel type.

\section{Discussion}

\section{Replicative capacity in vitro versus age of donor}

For both cell types there are fewer data points for the younger ages of 0 to 30 years, which is to be expected, as there is less availability for vessel biopsies (with the exception of HUVECs). Comparing the graph of the VSMCs with those of the ECs, the observed trends and average CPD values per age are roughly similar between the two cell types.

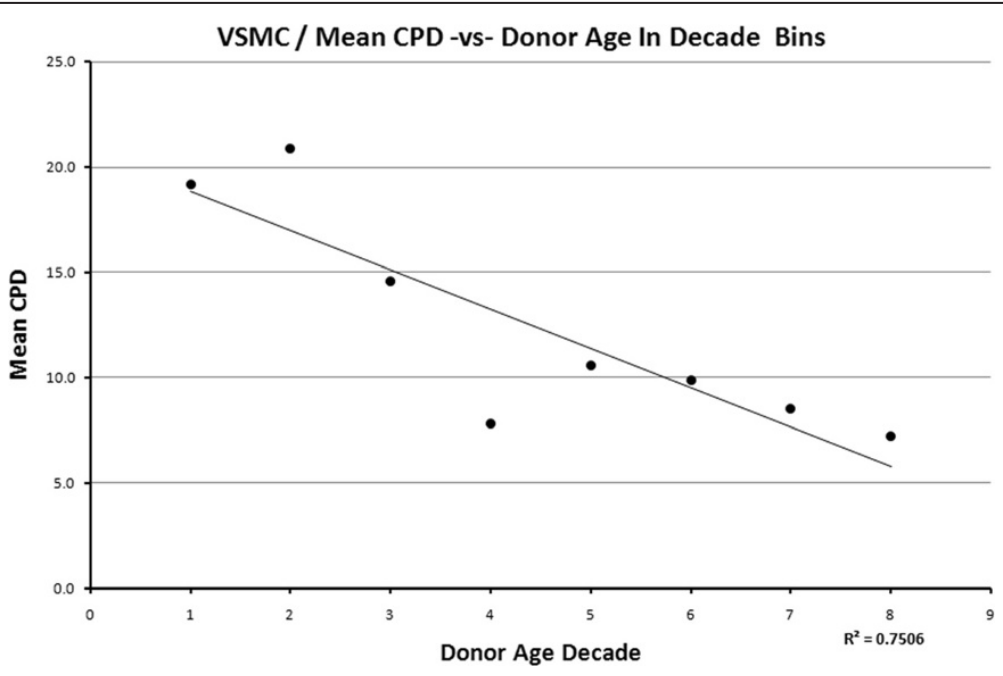

Figure 4 Relationship between the decade mean CPDs and donor age decade in vascular smooth muscle cells. 
Table 1 Table showing cell type, decades being compared, $P$-values and T-test interpretation

\begin{tabular}{|c|c|c|c|}
\hline Cell type & Decades being compared & $P$-value & T-test interpretation \\
\hline VSMC & 1,2 & 0.734 & No Sig. Diff. \\
\hline VSMC & 2,3 & 0.386 & No Sig. Diff. \\
\hline VSMC & 3,4 & 0.393 & No Sig. Diff. \\
\hline VSMC & 4,5 & 0.764 & No Sig. Diff. \\
\hline VSMC & 5,6 & 0.856 & No Sig. Diff. \\
\hline VSMC & 6,7 & 0.597 & No Sig. Diff. \\
\hline VSMC & 7,8 & 0.638 & No Sig. Diff. \\
\hline VSMC & 1,8 & 3.47E-04 & Sig. Diff. \\
\hline VSMC & $1+2,7+8$ & $1.43 \mathrm{E}-05$ & Sig. Diff. \\
\hline EC (all) & 1,2 & 0.0205 & Sig. Diff. \\
\hline EC (all) & 2,3 & 0.715 & No Sig. Diff. \\
\hline EC (all) & 3,4 & 0.947 & No Sig. Diff. \\
\hline EC (all) & 4,5 & 0.0235 & Sig. Diff. \\
\hline EC (all) & 5,6 & 0.444 & No Sig. Diff. \\
\hline EC (all) & 6,7 & 0.838 & No Sig. Diff. \\
\hline EC (all) & 7,8 & 0.887 & No Sig. Diff. \\
\hline EC (all) & 8,9 & 0.635 & No Sig. Diff. \\
\hline EC (all) & 1,8 & 4.04E-10 & Sig. Diff. \\
\hline EC (all) & $1+2+3,7+8+9$ & $5.46 \mathrm{E}-12$ & Sig. Diff. \\
\hline EC (excluding HUVECs) & $2+3,7+8+9$ & 1.98E-03 & Sig. Diff. \\
\hline
\end{tabular}

The data presented here are consistent with previous findings, showing a definite decline in proliferative activity in vitro with donor age. If the mean decade CPD values are used (to balance out the effects of possible confounding factors), the change in CPDs with age of both cell types corresponds to the finding by Martin, et al. [6] of approximately two CPDs per decade in fibroblasts. The findings are also strongly consistent with Hayflick's observations on fibroblasts (1965) [33]; ECs derived from human embryos underwent about 48 (mean average) CPDs in vitro, ranging from 18 to 79 CPDs, while ECs derived from adults underwent roughly 20 (mean average) CPDs, ranging from 3 to 30 CPDs (excluding the two extreme outliers). Hayflick and Martin also emphasized the lack of correlation in the proliferative ability of cells derived from individuals of intermediate ages, despite the clear difference in the proliferative ability of cells derived from very old (70+)

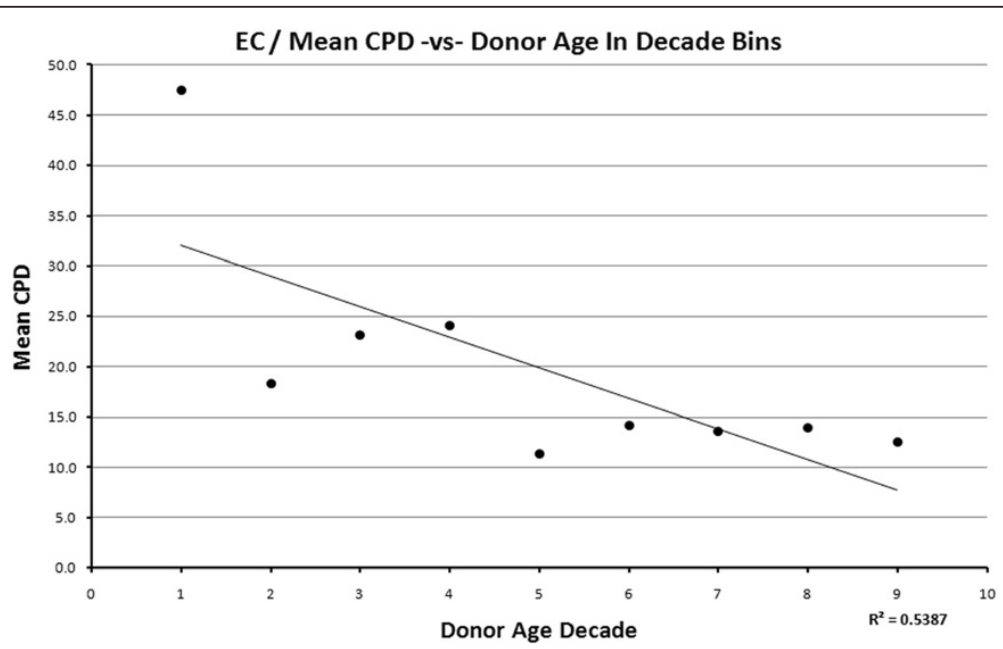

Figure 5 Relationship between the decade mean CPDs and donor age decade in endothelial cells. 
Table 2 Table showing cell type, variables and change in cumulative population doublings per year

\begin{tabular}{lll}
\hline Cell type & Variables & Change in CPD per year \\
\hline EC & CPD values -vs-donor age (all) & -0.44 \\
EC & CPD values-vs-donor age (excluding HUVECs) & -0.15 \\
EC & Mean CPD values-vs-donor age decade (all) & -0.19 \\
EC & Mean CPD values-vs-donor age decade (excluding HUVECs) & -0.19 \\
VSMC & CPD values -vs-donor age & -0.19 \\
VSMC & Mean CPD values-vs-donor age decade & -0.19 \\
EC & CPD -vs- donor age in donors with cardiovascular-related diseases (all) & -0.14 \\
EC & CPD -vs- donor age in donors without cardiovascular-related diseases (all) & -0.47 \\
EC & CPD -vs- donor age in donors without cardiovascular-related diseases (excluding HUVECs) & -0.16 \\
VSMC & CPD -vs- donor age in donors with cardiovascular-related diseases & -0.17 \\
VSMC & CPD -vs- donor age in donors without cardiovascular-related diseases & -0.2 \\
\hline
\end{tabular}

or very young (0 to 10$)$ donors. The same conclusions were drawn in this study, as demonstrated by the T-test calculations between adjacent and extreme ages. The exception to this is the difference in CPDs between decades 4 and 5 in ECs. The graph shows a very clear and sudden decrease in replicative capacity between the two decades and the $P$-value shows a significant difference between the two datasets. A reason for this may be that the decline in replicative capacity as a function of age may not occur at a consistent rate, possibly showing an abrupt decrease at certain points in the lifetime. The relationship between in vitro replicative capacity and organismal ageing should, therefore, not be interpreted to be linear or very tight [34].

\section{Replicative capacity in vitro versus age of donor, with} respect to cardiovascular-related diseases

Many studies on vascular smooth muscle cells and endothelial cells have reported a relationship between the altered cellular phenotype associated with senescence and the onset of cardiovascular disease [18-21,35,36]. In this review, the graphs show a vivid relationship between cardiovascular-related disease and replicative capacity in vascular cells, supported by the T-test calculations. It is clear from the graphs that individuals with cardiovascular-disease show a lower cellular proliferative potential than those without cardiovascular disease. This suggests that disease state is more strongly associated with the onset of senescence than donor age.

Table 3 Table showing cell type, publication, change in CPD per year, and number of samples

\begin{tabular}{llll}
\hline Cell type & Variables & Change in CPD per year & No. of samples \\
\hline VSMC & Eskin (1981) [47] & -0.029 & 64 \\
VSMC & Fukai (1994) [42] & -0.168 & 12 \\
VSMC & Kan (1987) [48] & -0.176 & 4 \\
VSMC & Bierman (1978) [49] & -0.03 & 17 \\
EC & Hoshi (1986) [50] & -0.206 & 8 \\
EC & Glassberg (1982) [51] & 0 & 4 \\
EC & Johnson (1992) [52] & -0.216 & 11 \\
EC & Maciag (1981) [53] & NA & 4 \\
EC & Nobuhiko (1988) [54] & NA & 2 \\
EC & Vogel (2008) [37] & NA & 2 \\
EC & Watkins (1993) [41] & +0.45 & 2 \\
EC & Vogel (2007) [55] & +0.119 & 61 \\
VSMC & TOTAL & $-\mathbf{0 . 1 9}$ & $\mathbf{1 0 7}$ \\
EC & TOTAL & -0.44 & $\mathbf{1 1 3}$ \\
\hline
\end{tabular}




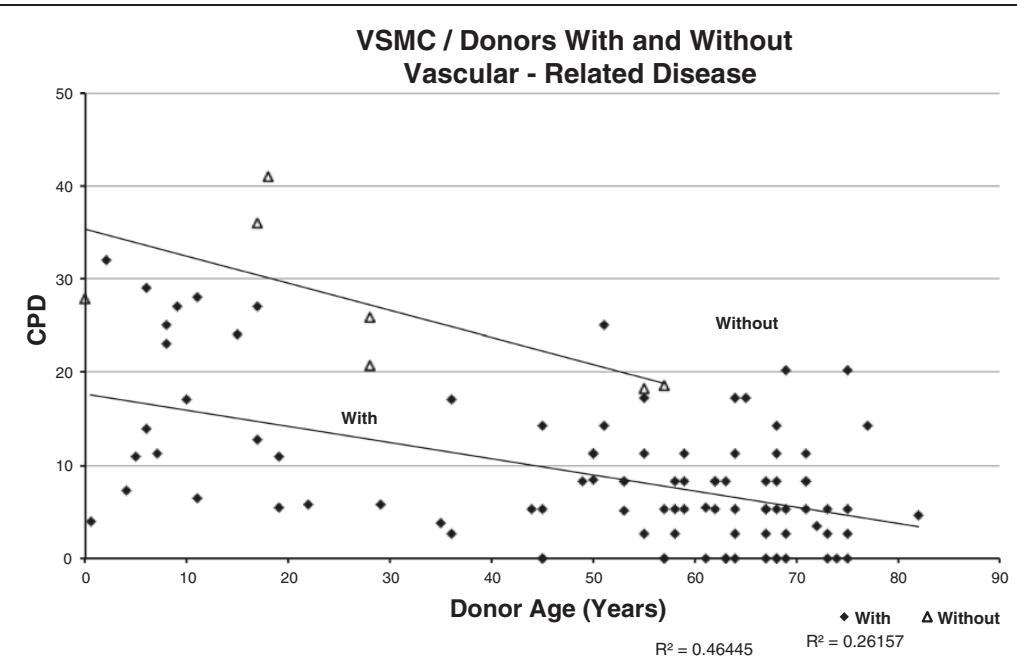

Figure 6 Relationship between CPDs and donor age in VSMCs, showing donors with and without cardiovascular-related diseases.

Looking at the replicative capacity for HUVECs alone (limited data for embryo-derived VSMCs), the extremely wide scatter ranging from 18 to 79 CPDs already implies a variation in terms of inherited proliferative capacity, possibly attributed to differences in telomere length. Inherited telomere length is, in turn, thought to be affected by paternal age at reproduction [37]. If replicative capacity can be considered a heritable trait, it follows that neonates showing inherent low cellular proliferative capacity will be more prone to disease later in life. Since neonates cannot be distinguished on the basis of liability to disease (and because none are yet diagnosed with disease), including these in the graphical relation between disease and replicative capacity distorts the trend; classing all HUVECs as without disease is misleading, as these will show the trait assumed to underlie disease predisposition (low replicative capacity), as do the adult donors with disease.
When HUVECs are excluded, both ECs and VSMCs show that diseased individuals start off with a lower replicative capacity than non-diseased individuals. The difference in average CPDs in age-matched individuals in the two groups is roughly constant throughout the lifetime, that is, an average difference of 7 CPDs in ECs and 20 CPDs in VSMCs. This essentially supports the hypothesis suggesting that individuals differ in terms of inherited cellular replicative capacity from the start of their lifespan, but exhaust this proliferative potential at similar rates. These conclusions are in line with the assumption that inter-individual inherited telomere length varies widely. Substantial variations of telomere lengths have been reported in some studies [29,30,32]; however, the focus has been on adult donors. In adults, telomere shortening may occur following reparative cell divisions, exposure to stress or early infection, as well as differences in inherited telomere lengths. This probably

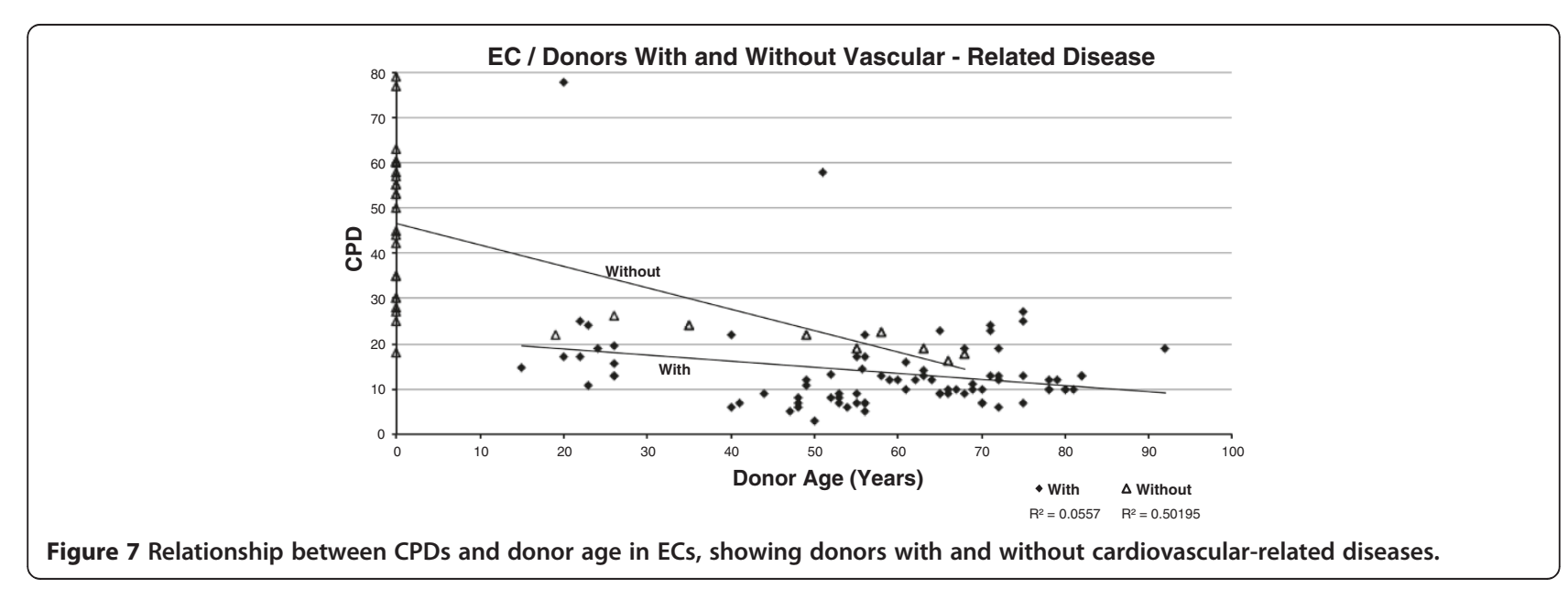




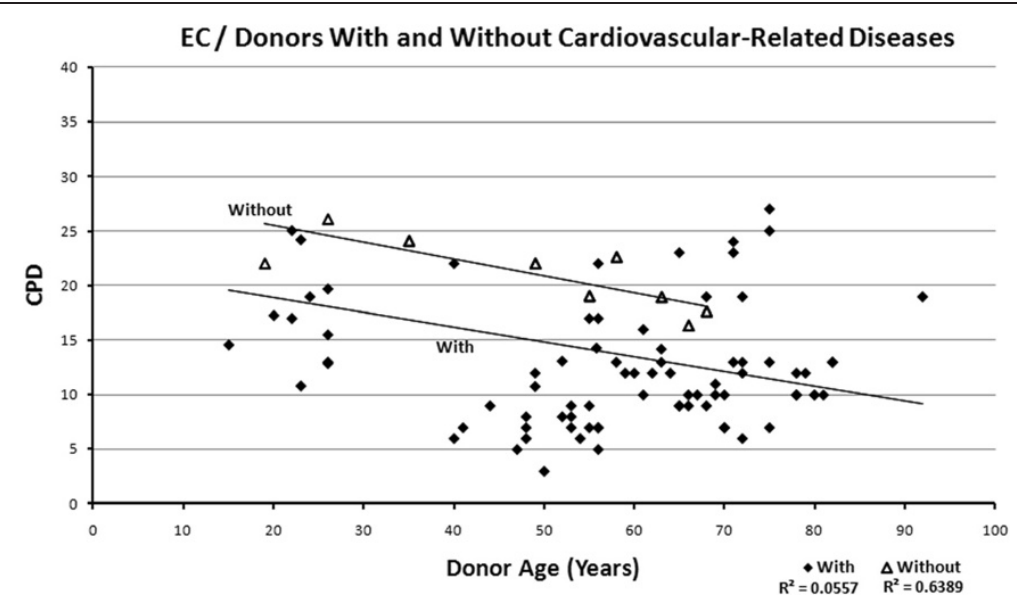

Figure 8 Relationship between CPDs and donor age in ECs, showing donors with and without cardiovascular-related diseases, excluding HUVEC data points.

accounts for the scattered distribution of data points corresponding to adult donors observed in this analysis. It would be more interesting if variation in telomere length of cells derived from human embryos were investigated in association with paternal age and telomere length in sperm. As far as we know, population variations of telomere length in neonates (that is, HUVECs) and with respect to paternal characteristics, has not yet been studied. Further exploration to elucidate the extent of variation of inherited telomere length and the possible consequences for disease, as initially implied in this review, is necessary. Despite the obvious inclination of these data toward inherited proliferative activity being strongly associated with disease state, the effect of decline in replicative capacity throughout the lifetime (that is, exogenous stress) should not be ignored. Cells from individuals with inherited short telomeres that are also exposed to considerable

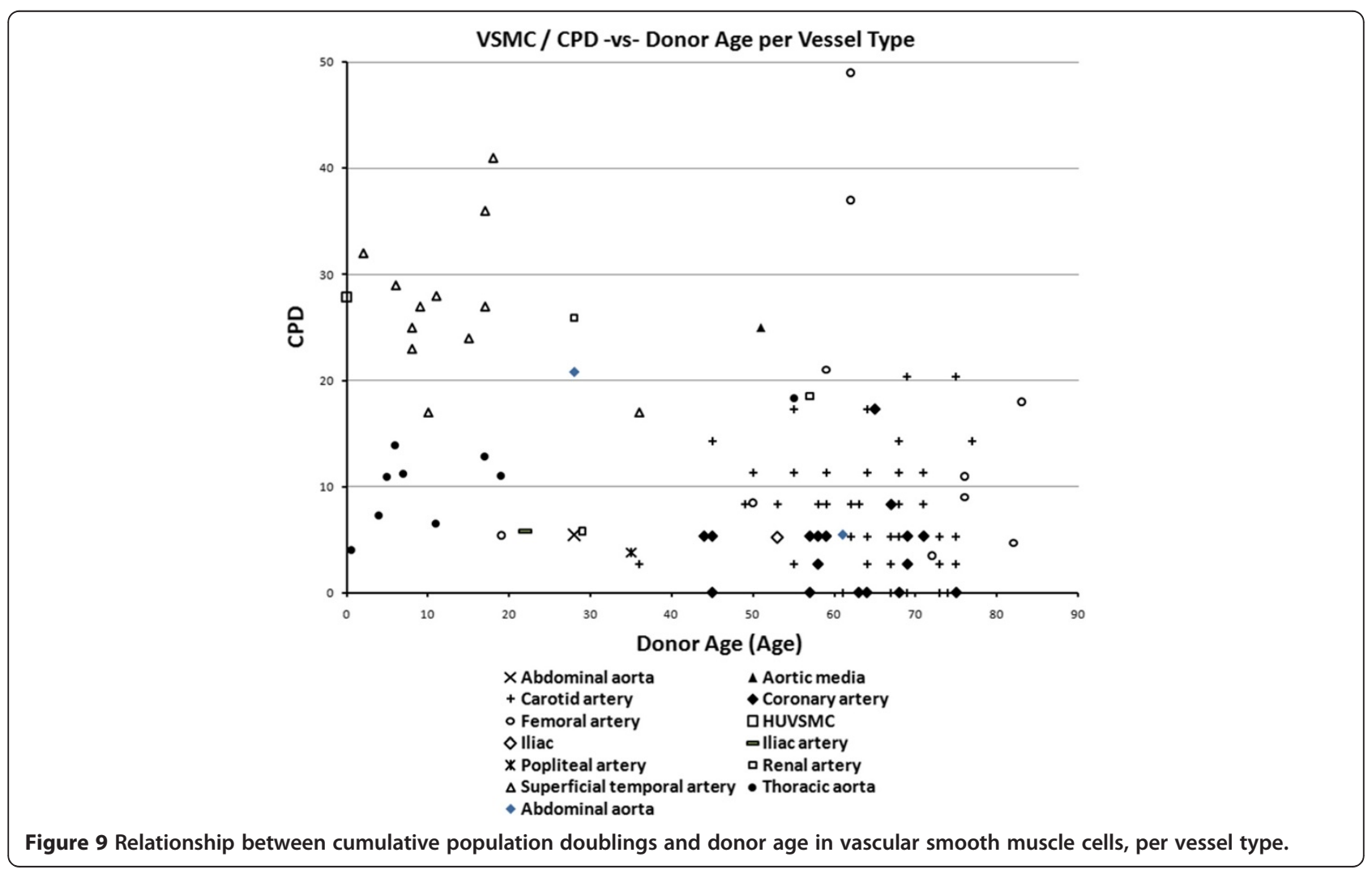




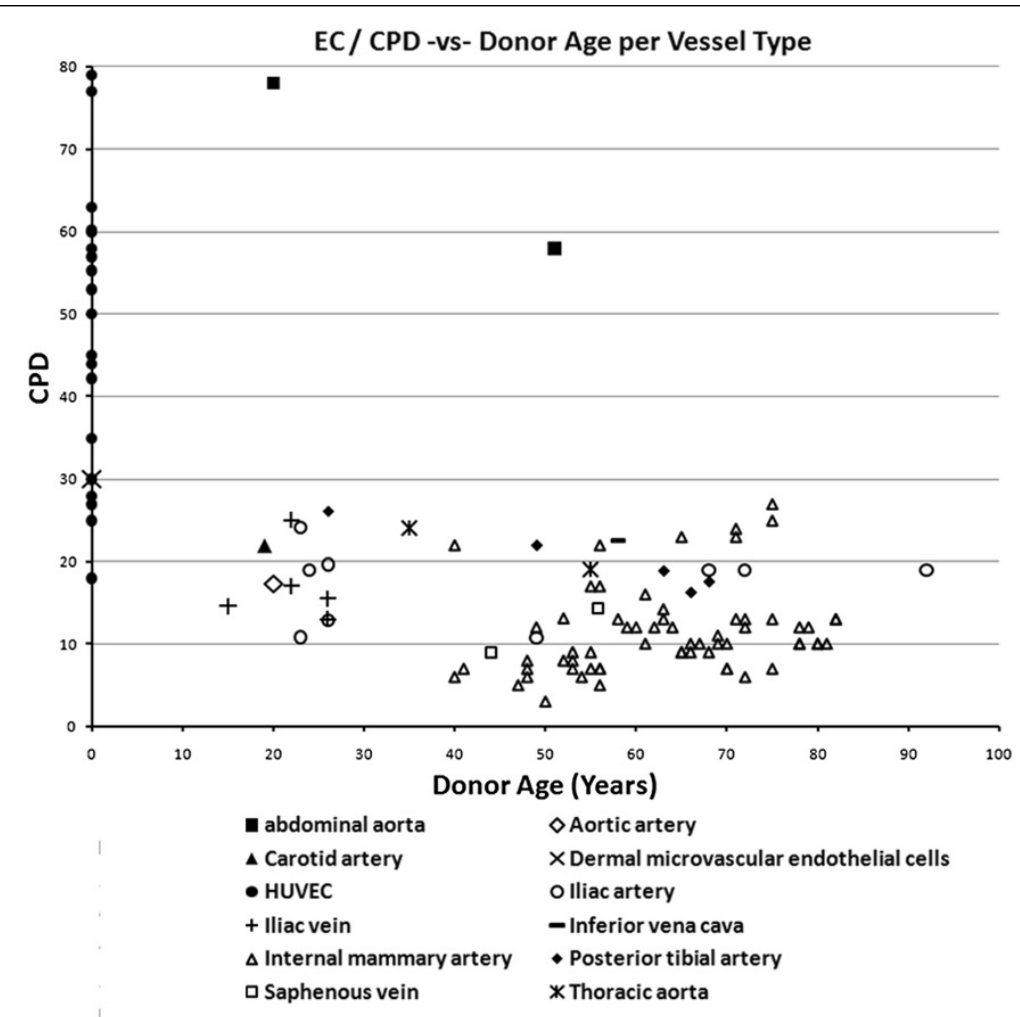

Figure 10 Relationship between cumulative population doublings and donor age in endothelial cells, per vessel type.

amounts of stress throughout the life course should undoubtedly show an even greater decrease in replicative capacity compared to healthy individuals.

While there is evidence that telomere shortening controls senescence in endothelial cells $[3,38]$, it is unclear whether vascular smooth muscle cells undergo telomere-dependent senescence. It has been demonstrated that in VSMCs telomerase reverse transcriptase (TERT) is regulated at the transcriptional level, it is unclear whether over-expression of telomerase can bypass senescence $[3,39]$. There are limitations in terms of experimental approaches in these studies: each of these experiments involved analysis of pooled colonies, which is unreflective, since replicative capacity of cells in culture reflects the expansive propagation of the longest surviving clone. A more informative approach should involve isolation of as many colonies of

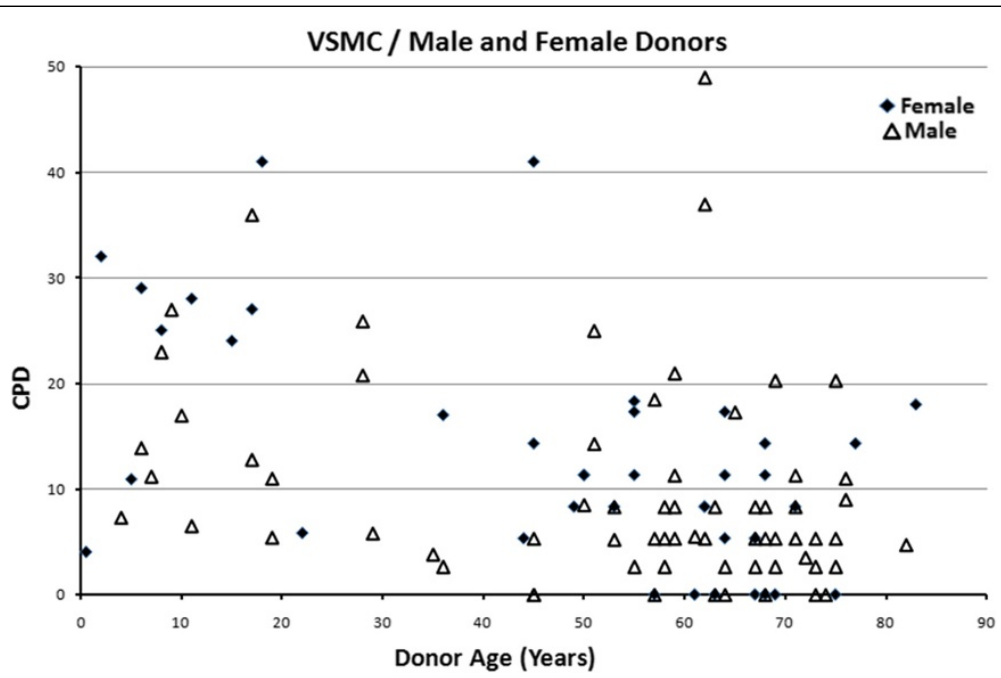

Figure 11 Relationship between cumulative population doublings and donor age in vascular smooth muscle cells, per gender. 


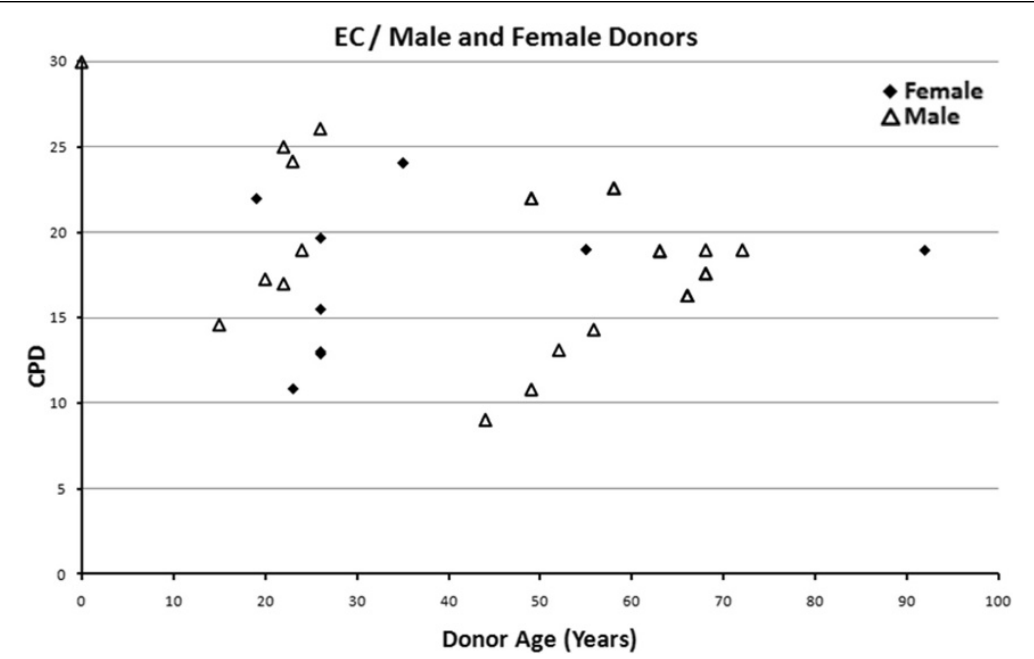

Figure 12 Relationship between cumulative population doublings and donor age in endothelial cells, per gender.

vector-infected cells as possible (if possible, approximately $\mathrm{n}=15$ ). If all the isolated clones are found to be immortalized, the cell type can be described with a high certainty to have undergone telomerase-induced escape from senescence.

\section{Limitations of this study}

A quantitative relationship between proliferative capacity and age cannot be made with absolute certainty from these data. Nevertheless, the correlations found are surprisingly good, given that the studies included span over 30 years, with possible differences in donor characteristics, cell culture techniques, growth media, explanation methods and selection of biopsy areas.

Growth media are also well-recognized variables affecting cell growth and proliferation. Varying concentrations of serum used in culture result in different growth effects, as demonstrated by Holley et al. [40], with the number of PDs achieved directly related to the original concentration of serum added. Studies varied in terms of serum type and concentration used; for example, EC were cultured with $30 \%$ human serum in the study by Watkins et al. [41] while $15 \%$ fetal bovine serum was used in the study by Fukai et al. [42].

Studies also varied in terms of their definition of senescence, ranging from: senescence-associated morphological changes, $50 \%$ positive for SA-beta-galactosidase staining, or less than one PD occurring within three weeks after subculture. Diverse interpretations of ceased proliferation may have led to inconsistencies of the recorded replicative capacity.

Vessel type is an additional variable known to affect cellular replicative ability; for example, telomere length has been reported to decrease more rapidly in arterial rather than venous endothelial cells [15]. It has even been shown that the segment of the vessel used affects telomere attrition; for example, the distal versus the proximal segment of the abdominal aorta showing accelerated telomere attrition [16]. This most likely reflects the hemodynamic stress factor [43-46].

The scatter plots showing the effect of vessel type and gender showed the data-points to be generally equally distributed. Any exceptions were most likely due to factors associated with disease, shear stress or age, as different studies varied in terms of sample characteristics.

It would be misleading to perform a Funnel test (to test for publication bias) or a Cochrane-Q test (to test for heterogeneity between studies included). Different researchers focused on specific age ranges or disease states; therefore, any asymmetry observed in a funnel plot will probably not be due to systematic error or publication bias, but be due to factors associated with disease, age or sheer stress.

It should also be considered that studies included in this analysis did not always specify the site from which the cells were explanted. Moreover, the removal of vascular tissue is much less likely to occur in healthy individuals, compared to fibroblasts (which are far easier to obtain), unless the donor had died in an accident. It is, therefore, uncertain that all of the donors classed as 'without cardiovascular-related disease' included in this study were entirely healthy. For example, it is possible that senescence in endothelial or vascular smooth muscle cells is also dependent on damage induced by other, unrelated pathological conditions in the individual. 
Despite all of this, the plots still show a clear inverse relationship between donor age and proliferative capacity in vitro. These conclusions are supported by the T-test values and correlation coefficients.

\section{Conclusions}

This survey of the available literature demonstrates that a clear inverse relationship exists between the replicative capacity of vascular endothelial and smooth muscle cells and donor age. Individuals free of cardiovascular-related diseases show a greater replicative capacity at given ages, than those with defined pathologies. The mean CPD for each diseased age group roughly corresponds to that of older, non-diseased subjects. This suggests that disease state rather than donor age is the primary variable correlating with senescence. Perhaps surprisingly, individuals with cardiovascular disease appear to start off with a lower cellular replicative capacity in early life, than non-diseased individuals. The difference in average CPDs in age-matched individuals in the two groups is roughly constant throughout the lifetime. This is consistent with the hypothesis that individuals differ in terms of inherited cellular replicative capacity, but exhaust their proliferative potential at similar rates.

\section{Abbreviations}

CPD: Cumulative Population Doublings; DNA: Deoxyribonucleic acid; EC: Endothelial Cell; HUVEC: Human Umbilical Vein Endothelial Cells; IL: 1aInterleukin-1 alpha; ICAM-1: Intracellular Adhesion Molecule 1; NA: Not Applicable; PAI-1: Plasminogen Activator Inhibitor-1; TERT: Telomerase Reverse Transcriptase; VSMC: Vascular Smooth Muscle Cell.

\section{Competing interests}

The authors declare that they have no competing interests.

\section{Authors' contributions}

RF devised, planned and assigned this project to MK to satisfy the criteria of completing a BSc. MK collected and analyzed the data and drafted the manuscript under the supervision of RF. All authors read and approved the final manuscript.

\section{Author details}

'School of Medicine, The Commonwealth Building, The Hammersmith Hospital, Imperial College London, Du Cane Road, London W12 ONN, UK. ${ }^{2} \mathrm{School}$ of Pharmacy and Biomolecular Sciences, University of Brighton, Huxley Building, Lewes Road, Brighton BN2 4GJ, UK.

Received: 23 January 2012 Accepted: 12 November 2012 Published: 4 February 2013

\section{References}

1. Yang DG, Liu L, Zheng XY: Cyclin-dependent kinase inhibitor p16(INK4a) and telomerase may co-modulate endothelial progenitor cells senescence. Ageing Res Rev 2008, 7:137-146.

2. Minamino T, Komuro I: Vascular aging: insights from studies on cellular senescence, stem cell aging, and progeroid syndromes. Nat Clin Pract Cardiovasc Med 2008, 5:637-648.

3. Matthews C, Gorenne I, Scott S, Figg N, Kirkpatrick P, Ritchie A, Goddard M Bennett M: Vascular smooth muscle cells undergo telomere-based senescence in human atherosclerosis: effects of telomerase and oxidative stress. Circ Res 2006, 99:156-164.

4. Burton DG, Giles PJ, Sheerin AN, Smith SK, Lawton JJ, Ostler EL, RhysWilliams W, Kipling D, Faragher RG: Microarray analysis of senescent vascular smooth muscle cells: a link to atherosclerosis and vascular calcification. Exp Gerontol 2009, 44:659-665.

5. Baker DJ, Wijshake T, Tchkonia T, LeBrasseur NK, Childs BG, van de Sluis B, Kirkland $J$, van Deursen JM: Clearance of p16Ink4a-positive senescent cells delays ageing-associated disorders. Nature 2011, 479:232-236.

6. Martin GM, Sprague CA, Epstein CJ: Replicative lifespan of cultivated human cells. Effects of donor's age, tissue and genotype. Lab Invest 1970, 23:86-92.

7. Schneider EL, Mitsui Y: The relationship between in vitro cellular aging and in vivo human age. Proc Natl Acad Sci U S A 1976, 73:3584-3588.

8. Goldstein S, Moerman EJ, Soeldner JS, Gleason RE, Barnett DM: Chronologic and physiologic age affect replicative life-span of fibroblasts from diabetic, prediabetic, and normal donors. Science 1978, 199:781-782.

9. Cristofalo VJ, Allen RG, Pignolo RJ, Martin BG, Beck JC: Relationship between donor age and the replicative lifespan of human cells in culture: a reevaluation. Proc Natl Acad Sci U S A 1998, 95:10614-10619.

10. Maier $A B$, Westendorp RG: Relation between replicative senescence of human fibroblasts and life history characteristics. Ageing Res Rev 2009, 8:237-243

11. Maier AB, le Cessie S, de Koning-Treurniet C, Blom J, Westendorp RG, van Heemst D: Persistence of high-replicative capacity in cultured fibroblasts from nonagerians. Aging Cell 2007, 6:27-33.

12. Dimri GP, Lee X, Basile G, Acosta M, Scott G, Roskelley C, Medrano EE, Linskens M, Rubelj I, Pereira-Smith $\mathrm{O}$, et al: A biomarker that identifies senescent human cells in culture and in aging skin in vivo. Proc Natl Acad Sci U S A 1995, 92:9363-9367.

13. Herbig U, Ferreira M, Condel L, Carey D, Sedivy JM: Cellular senescence in aging primates. Science 2006, 311:1257.

14. Li Y, Yan Q, Wolf NS: Long-term caloric restriction delays age-related decline in proliferation capacity of murine lens epithelial cells in vitro and in vivo. Invest Ophthalmol Vis Sci 1997, 38:100-107.

15. Fenton M, Barker S, Kurz DJ, Erusalimsky JD: Cellular senescence after single and repeated balloon catheter denudations of rabbit carotid arteries. Arterioscler Thromb Vasc Biol 2001, 21:220-226.

16. Aviv H, Khan MY, Skurnick J, Okuda K, Kimura M, Gardner J, Priolo L, Aviv A: Age dependent aneuploidy and telomere length of the human vascular endothelium. Atherosclerosis 2001, 159:281-287.

17. Bennet MR, Evan Gl, Schwartz SM: Apoptosis of human vascular smooth muscle cells derived from normal vessels and coronary atherosclerotic plaques. J Clin Invest 1995, 95:2266-2274.

18. Moyer CF, Sajuthi D, Tulli H, Williams JK: Synthesis of IL-1 alpha and IL-1 beta by arterial cells in atherosclerosis. Am J Pathol 1991, 138:951-960.

19. Schneiderman J, Sawdey MS, Keeton MR, Bordin GM, Bernstein EF, Dilley RB, Loskutoff DJ: Increased type 1 plasminogen activator inhibitor gene expression in atherosclerotic human arteries. Proc Natl Acad Sci U S A 1992, 89:6998-7002.

20. van der Wal AC, Das PK, Tigges AJ, Becker AE: Adhesion molecules on the endothelium and mononuclear cells in human atherosclerotic lesions. Am J Pathol 1992, 141:1427-1433.

21. Burton DG, Matsubara $H$, Ikeda K: Pathophysiology of vascular calcification: Pivotal role of cellular senescence in vascular smooth muscle cells. Exp Gerontol 2010, 45:819-824.

22. Chang E, Harley CB: Telomere length and replicative aging in human vascular tissues. Proc Natl Acad Sci U S A 1995, 92:11190-11194.

23. Slagboom PE, Droog S, Boomsma DI: Genetic determination of telomere size in humans: a twin study of three age groups. Am J Hum Genet 1994, 55:876-882

24. Brouilette SW, Moore JS, MCMahon AD, Thompson JR, Ford I, Shepherd J, Packard CJ, Samani NJ, West of Scotland Coronary Prevention Study Group: Telomere length, risk of coronary heart disease, and statin treatment in the West of Scotland Primary Prevention Study: a nested case-control study. Lancet 2007, 369:107-114.

25. Cawthon R, Smith KR, O'Brien E, Sivatchenko A, Kerber RA: Association between telomere length in blood and mortality in people aged 60 years or older. Lancet 2003, 361:393-395.

26. Ben-Porath I, Weinberg RA: The signals and pathways activating cellular senescence. Int J Biochem Cell Biol 2005, 37:961-976.

27. Toussaint O, Medrano EE, von Zglinicki T: Cellular and molecular mechanisms of stress-induced premature senescence (SIPS) of human diploid fibroblasts and melanocytes. Exp Gerontol 2000, 35:927-945.

28. Eisenberg DT, Salpea KD, Kuzawa CW, Hayes MG, Humphries SE, European Atherosclerosis Research Study II Group: Substantial variation in qPCR 
measured mean blood telomere lengths in young men from eleven European countries. Am J Hum Biol 2011, 23:228-231.

29. Hunt SC, Chen W, Gardner JP, Kimura M, Srinivasan SR, Eckfeldt JH, Berenson GS, Aviv A: Leukocyte telomeres are longer in African Americans than in whites: the National Heart, Lung, and Blood Institute Family Heart Study and the Bogalusa Heart Study. Aging Cell 2008, 7:451-458.

30. Demerath E, Cameron N, Gillman MW, Towne B, Siervogel RM: Telomeres and telomerase in the fetal origins of cardiovascular disease: a review. Hum Biol 2004, 76:127-146.

31. Epel ES, Blackburn EH, Lin J, Dhabhar FS, Adler NE, Morrow JD, Cawthon RM: Accelerated telomere shortening in response to life stress. Proc Nat Acad Sci U S A 2004, 101:17312-17315.

32. Kimura M, Cherkas LF, Kato BS, Demissie S, Hjelmborg JB, Brimacombe $M$, Cupples A, Hunkin JL, Gardner JP, Lu X, Cao X, Sastrasinh M, Province MA, Hunt SC, Christensen K, Levy D, Spector TD, Aviv A: Offspring's Leukocyte telomere length, paternal age, and telomere elongation in sperm. PLoS Genet 2008, 4:e37.

33. Hayflick L: The limited in vitro lifetime of human diploid cell strains. Exp Cell Res 1965, 37:614-636

34. Arking R: The Biology of Aging: Observations and Principles. 2nd edition. Sunderland, MA, USA: Sinauer Associates, Inc; 1998.

35. Nakano-Kurimoto R, Ikeda K, Uraoka M, Nakagawa Y, Yutaka K, Koide M, Takahashi T, Matoba S, Yamada H, Okigaki M, Matsubara H: Replicative senescence of vascular smooth muscle cells enhances the calcification through initiating the osteoblastic transition. Am J Physiol Heart Circ Physiol 2009, 297:H1673-1684.

36. Hampel B, Fortschegger $\mathrm{K}$, Ressler $\mathrm{S}$, Chang MW, Unterluggauer $\mathrm{H}_{\text {, }}$ Breitwieser A, Sommergruber W, Fitzky B, Lepperdinger G, Jansen-Dürr P, Voglauer R, Grillari J: Increased expression of extracellular proteins as a hallmark of human endothelial cell in vitro senescence. Exp Gerontol 2006, 41:474-481.

37. Vogel G, Thorin-Trescases N, Farhat N, Nguyen A, Villeneuve L, Mamarbach AM, Fortier A, Perrault LP, Carrier M, Thorin E: Cellular senescence in endothelial cells from atherosclerotic patients is acceleration by oxidative stress associated with cardiovascular risk factors. Mech Ageing Dev 2007, 128:662-671.

38. Hastings R, Qureshi M, Verma R, Lacy PS, Williams B: Telomere attrition and accumulation of senescent cells in cultured human endothelial cells. Cell Prolif 2004, 37:317-324

39. Garton KJ, Ferri N, Raines EW: Efficient expression of exogenous genes in primary vascular cells using IRES-based retroviral vectors. Biotechniques 2002, 32:830-834.

40. Holley RW, Kiernan JA: Control of the initiation of DNA synthesis in 3T3 cells: Low-molecular-weight nutrients. Proc Nat Acad Sci U S A 1974, 71:2942-2945

41. Watkins MT, Sharefkin JB, Zajtchuk R, Maciag TM, D'Amore PA, Ryan US, Van Wart $H$, Rich NM: Adult human saphenous vein endothelial cells: assessment of their reproductive capacity for Use in endothelial seeding of vascular prostheses. J Surg Res 1983, 36:588-596.

42. Fukai N, Aoyagi M, Yamamoto M, Sakamoto H, Ogami K, Matsushima Y, Yamamoto K: Human arterial smooth muscle cell strains derived from patients with moyamoya disease: changes in biological characteristics and proliferative response during cellular ageing in vitro. Mech Ageing Dev 1994, 75:21-33.

43. Roach MR, Smith NB: Does high shear stress induced by blood flow lead to atherosclerosis? Perspect Biol Med 1983, 26:287-303.

44. Nerem RM, Levesque MJ: Fluid dynamics as a factor in the localization of atherogenesis. Ann N Y Acad Sci 1983, 416:709-710.

45. Davies PF: Hemodynamic shear stress and the endothelium in cardiovascular pathophysiology. Nat Clin Pract Cardiovasc Med 2009, 6:16-26.

46. Okuda K, Khan MY, Skurnick J, Kimura M, Aviv H, Aviv A: Telomere attrition of the human abdominal aorta: relationships with age and atherosclerosis. Atherosclerosis 2000, 152:391-398.

47. Eskin SG, Sybers HD, Lester JW, Navarro LT, Gotto AM, DeBakey ME: Human smooth muscle cells cultured from atherosclerotic plaques and uninvolved vessel wall. In Vitro 1981, 17:713-718.

48. Hoshi H, Kan M, Chen J, McKeehan W: Comparative endocrinologyparacrinology-autocrinology of human adult large vessel endothelial and smooth muscle cells. In Vitro Cell Dev Biol 1987, 24:309-320.

49. Bierman EL: The effect of donor Age on the in vitro life span of cultured human arterial smooth-muscle cells. In Vitro 1978, 14:951-955.
50. Hoshi H, McKeehan WL: Isolation, growth requirements, cloning, prostacyclin production and life-span of human adult endothelial cells in low serum culture medium. In Vitro Cell Dev Biol 1986, 22:51-56.

51. Glassberg MK, Bern MM, Coughlin SR, Haudenschild CC, Hoyer L, Antoniades HN, Zetter BR: Cultured endothelial cells derived from the human iliac arteries. In Vitro 1982, 18:859-866.

52. Johnson TE, Umbenhauer DR, Hill R, Bradt C, Mueller SN, Levine EM, Nichols WW: Karyotypic and phenotypic changes during in vitro aging of human endothelial cells. J Cell Physiol 1992, 150:17-27.

53. Maciag T, Hoover GA, Stemerman MB, Weinstein R: Serial propagation of human endothelial cells in vitro. J Cell Biol 1981, 91:420-426.

54. Nobuhiko H, Yamamoto M, Imamura T, Mitsui $Y$, Yamamoto K: Evaluation of long-term cultured endothelial cells as a model system for studying vascular ageing. Mech Ageing Dev 1988, 46:111-123.

55. Vogel G, Thorin-Trescases N, Farhat N, Mamarbachi AM, Villeneuve L, Fortier A, Perrault LP, Carrier M, Thorin E: Chronic treatment with $\mathrm{N}$-acetyl-cystein delays cellular senescence in endothelial cells isolated from a subgroup of atherosclerotic patients. Mech Ageing Dev 2008, 129:261-270.

doi:10.1186/2046-2395-2-3

Cite this article as: Karavassilis and Faragher: A relationship exists between replicative senescence and cardiovascular health. Longevity \& Healthspan 2013 2:3.

\section{Submit your next manuscript to BioMed Central and take full advantage of:}

- Convenient online submission

- Thorough peer review

- No space constraints or color figure charges

- Immediate publication on acceptance

- Inclusion in PubMed, CAS, Scopus and Google Scholar

- Research which is freely available for redistribution

Submit your manuscript at www.biomedcentral.com/submit
C Biomed Central 